\title{
An Statistical Filtering Models Comparison for GNSS LEO Satellite Navigation
}

\author{
Jorge Cogo*, Javier G. García*, Pedro A. Roncagliolo* and Carlos H. Muravchik*†‡ \\ * Departamento de Electrotecnia, Facultad de Ingeniería, Universidad Nacional de La Plata (UNLP). \\ Calle 48 y 116, La Plata, Bs. As., Argentina. \\ $\dagger$ Instituto LEICI, UNLP. Calle 48 y 116, La Plata, Bs. As., Argentina. \\ ${ }^{\ddagger}$ CIC-PBA. Calle 526 entre 10 y 11, La Plata, Bs. As., Argentina. \\ \{jorge.cogo, jgarcia, agustinr, carlosm\}@ing.unlp.edu.ar
}

\begin{abstract}
In this work the performance of different statistical filtering models used for estimating states of aerospace vehicles, particularly LEO satellites, based on measurements of GNSS systems are compared. This problem is non-linear in nature, since both the state variables model and the output function are non-linear. Thus we resort to the use of the extension of the Kalman filter called EKF.

Different models based on several kinematic and dynamic approaches are considered. For the performance assessment we use representative simulation scenarios. Finally, as a real application example, the case of GPS measurements taken on board the Argentine SAC-D satellite is analyzed.
\end{abstract}

\section{INTRODUCTION}

The Global Navigation Satellite Systems (GNSS) provide an alternative for the accurate determination (estimation) of position and velocity of Low Earth Orbit (LEO) satellites. Currently there are several fully operational GNSS systems as GPS (USA) and GLONASS (Russia), or in stage of development, as Galileo (European Union) and Beidou (China) [1], [2].

The measurements obtained with GNSS are related to the satellite-receiver range (pseudorange) and their rate of change (deltarange). The former is based on the signal time-of-arrival, while the latter is based on the carrier doppler frequency shift. Since it requires synchronism of time references of both the GNSS system and the user (receiver), which can't be ensured a-priori, two additional unknowns are incorporated to the problem: bias and drift of the user's clock. A mathematical model of the measurements is presented in section II.

These measurements are affected by various factors that induce both systematic and random errors. For the first type of errors one generally resorts to the use of models to discount or at least reduce them, while to mitigate the effect of the latter, statistical filtering methods are often used. These filtering methods usually take advantage of a model that describes vehicle behavior in terms of a state variable system.

To obtain state variable models for LEO satellite vehicles we use two approaches of classical physics: the kinematic approach and the dynamic approach. Kinematics studies the laws of motion of bodies without considering the causes that originate them. Such description generally begins with the knowledge of some of the involved variables, and the rest are obtained based on derivation/integration as required. The models obtained based on this approach are described in section III.

On the other hand, dynamics studies the laws of motion of bodies in relation to the causes that originated them, the intervening forces. The formulation known as Newtonian mechanics enables the description of the motion based on ordinary differential equations in cartesian coordinates; this will be sufficient for the vehicles we want to model. This approach is described in section IV.

Since these models are of a non-linear nature, and that measurements obtained with GNSS are also related nonlinearly with the states, the problem of state estimation is also nonlinear. In numerous papers such as [3] and [4] filtering schemes based on the Extended Kalman Filter (EKF) for precise orbit determination based on GPS signals are used, and in [5] and [6] the case of orbit determination using other filtering schemes such as the Unscented Kalman Filter (UKF) or Sigma-Point Kalman Filter are presented.

In a previous work [7] the performance of two different filtering schemes (EKF and Positioning Kalman Filter (PKF)) is compared for the case of using the same vehicle model. In this paper we will focus on analyzing the behavior of the same filtering scheme, the EKF, with different versions of the vehicle model. A brief summary of the EKF formulation is described in section $\mathrm{V}$, while section VI describes how the formulation for each one of these analyzed models results.

In section VII, the performance of EKF with the different proposed models is compared by simulation, while in section VIII an application example with actual GPS data from onboard argentine SAC-D satellite is presented. Finally, in section IX the conclusions are set.

\section{Measurements Model}

The pseudorange measurement at time $k$ obtained by a user at position $\mathbf{r}_{k}$ based upon the signal of the $j$-th satellite at position $\mathbf{s}_{k j}$ is given by

$$
\rho_{k j}=\left\|\mathbf{s}_{k j}-\mathbf{r}_{k}\right\|+b_{k}+\varepsilon_{k j}+\nu_{k j}
$$

where $b_{k}$ is the receiver's clock bias multiplied by the speed of light to be interpreted as range, and $\varepsilon_{k j}$ and $\nu_{j k}$ group the sistematic and stochastic errors, respectively. We model the latter as an i.i.d. gaussian process, with zero mean and variance 
$\sigma_{\rho}^{2}$. We denote with $\boldsymbol{\rho}_{k}$ the column vector that groups all the pseudorange measurements available at time $k$.

We also denote

$$
\mathbf{e}_{k j} \triangleq \frac{\mathbf{s}_{k j}-\mathbf{r}_{k}}{\left\|\mathbf{s}_{k j}-\mathbf{r}_{k}\right\|}
$$

the line-of-sight vector that points from the user's position to the $j$-th GNSS satellite. If the user and satellite velocity vectors are $\dot{\mathbf{r}}_{k}$ and $\dot{\mathbf{s}}_{k j}$, respectively, the deltarange measurement of that satellite results

$$
\dot{\rho}_{k j}=\mathbf{e}_{k j} \cdot\left(\dot{\mathbf{s}}_{k j}-\dot{\mathbf{r}}_{k}\right)+\dot{b}_{k}+\xi_{k j}+v_{k j}
$$

where $\dot{b}_{k}$ is the user's clock drift converted to velocity, and $\xi_{k j}$ and $v_{j k}$ group the sistematic and stochastic errors, respectively. We model the latter as an i.i.d. gaussian process, with zero mean and variance $\sigma_{\dot{\rho}}^{2}$. We denote with $\dot{\boldsymbol{\rho}}_{k}$ the column vector that groups all the deltarange measurements available at time $k$.

\section{KinEMATIC APPROACH}

If we consider as state variables the three position states and the three velocity states (contained in $\mathbf{x}_{k}$ ), and as a disturbance input the three acceleration states (contained in $\mathbf{a}_{k}$ ); and neglect higher order terms, a kinematic model discretized in time every $T$ seconds is

$$
\mathbf{x}_{k}=\left[\begin{array}{cc}
\mathbf{I}_{3 \times 3} & T \mathbf{I}_{3 \times 3} \\
\mathbf{0}_{3 \times 3} & \mathbf{I}_{3 \times 3}
\end{array}\right] \mathbf{x}_{k-1}+\left[\begin{array}{cc}
\frac{T^{2}}{2} & \mathbf{I}_{3 \times 3} \\
T & \mathbf{I}_{3 \times 3}
\end{array}\right] \mathbf{a}_{k-1} .
$$

If we also consider that the state vector includes the three acceleration states, and considering as a disturbance input the three jerk states (contained in $\mathbf{j}_{k}$ ); neglecting the higher order terms, the model is

$$
\begin{gathered}
\mathbf{x}_{k}=\left[\begin{array}{ccc}
\mathbf{I}_{3 \times 3} & T \mathbf{I}_{3 \times 3} & \frac{T^{2}}{2} \mathbf{I}_{3 \times 3} \\
\mathbf{0}_{3 \times 3} & \mathbf{I}_{3 \times 3} & T \\
\mathbf{0}_{3 \times 3} & \mathbf{0}_{3 \times 3} & \mathbf{I}_{3 \times 3}
\end{array}\right] \mathbf{x}_{k-1}+ \\
+\left[\begin{array}{cc}
\frac{T^{3}}{6} & \mathbf{I}_{3 \times 3} \\
\frac{T^{2}}{2} & \mathbf{I}_{3 \times 3} \\
T & \mathbf{I}_{3 \times 3}
\end{array}\right] \mathbf{j}_{k-1} .
\end{gathered}
$$

Equations 4 and 5 represent linear time-invariant discrete systems, in whose formulation no assumption was made about the causes of the acceleration (or higher order terms), in line with a kinematic approach. Therefore, these models can be used in a wide variety of vehicles when no other, more accurate model is available. Moreover, being very simple and timeinvariant models, they often result in a low computational load implementation.

\section{DYNAMIC APPROACH}

To describe the orbital motion from a dynamic approach, we start from the equation describing the acceleration of a satellite in the ECI frame (Earth-Centered Inertial) [8]

$$
\ddot{\mathbf{r}}=-\frac{\mu}{r^{3}} \mathbf{r}+\mathbf{a}_{p}
$$

where $\mu$ is the Earth's gravitational constant and $r=|\mathbf{r}|$. The first term corresponds to the effect of Earth's gravitational attraction, if it is modeled as a point mass, and $\mathbf{a}_{p}$ corresponds to the acceleration due to other factors. In the case of LEO satellites, the four effects that contribute the most to $\mathbf{a}_{p}$ are: non-uniform distribution of Earth's mass, $\mathbf{a}_{n e}$; gravitational attraction of other celestial bodies, $\mathbf{a}_{3 b}$; atmospheric drag, $\mathbf{a}_{d}$ and thrust of the engines used for orbital maneuvers, $\mathbf{a}_{t}[8]$

$$
\mathbf{a}_{p}=\mathbf{a}_{n e}+\mathbf{a}_{3 b}+\mathbf{a}_{d}+\mathbf{a}_{t} .
$$

To formulate Eq. 6 in an ECEF frame we need to add the terms due to the rotation between the ECEF and ECI frames [9]

$$
\ddot{\mathbf{r}}=-\frac{\mu}{r^{3}} \mathbf{r}+\mathbf{a}_{p}^{e}+2 \mathbf{S}\left(\boldsymbol{\omega}_{e i}^{e}\right) \dot{\mathbf{r}}+\mathbf{S}\left(\boldsymbol{\omega}_{e i}^{e}\right)^{2} \mathbf{r}
$$

where $\mathbf{a}_{p}^{e}$ denotes the acceleration due to disturbances expressed in the ECEF frame, $\mathbf{S}(\cdot)$ denotes the cross product matrix, $\mathbf{S}(\mathbf{x}) \mathbf{y}=\mathbf{x} \times \mathbf{y}$, and $\boldsymbol{\omega}_{e i}^{e}$ denotes the angular velocity vector of the ECI frame relative to the ECEF frame, measured in ECEF. In Eq. 8 the three vectors $\ddot{\mathbf{r}}, \dot{\mathbf{r}}$ and $\mathbf{r}$ are expressed in ECEF coordinates.

\section{KALMAN Filter (KF)}

The KF is an estimator for what is known as linear quadratic problem of estimating the state of a linear dynamic system disturbed by white noise, using measurements linearly related to the state and affected linearly by white noise. The resulting estimator is statistically optimal with respect to any quadratic function of the estimation error [10].

We will focus on analyzing the variant of the KF used for discrete systems, whose mathematical description is

$$
\begin{gathered}
\mathbf{x}_{k}=\mathbf{F}_{k-1} \mathbf{x}_{k-1}+\mathbf{G}_{k-1} \mathbf{w}_{k-1} \\
\mathbf{y}_{k}=\mathbf{H}_{k} \mathbf{x}_{k}+\mathbf{v}_{k}
\end{gathered}
$$

where $\mathbf{x}_{k}$ denotes the system's state, and $\mathbf{y}_{k}$ denotes the measurements vector, both at time $k$. Matrices $\mathbf{F}_{k-1}, \mathbf{G}_{k-1}$ and $\mathbf{H}_{k}$ are commonly referred to as System Matrix, Input Matrix and Output Matrix, respectively. The vectors $\mathbf{w}_{k}$ and $\mathbf{v}_{k}$ are modeled as uncorrelated white stochastic processes with zero mean and covariance matrices denoted $\mathbf{Q}_{k}$ and $\mathbf{R}_{k}$, respectively.

In the formulation of the KF, two estimates of the state vector are defined: $\hat{\mathbf{x}}_{k}^{-}$, or a-priori estimate, and $\hat{\mathbf{x}}_{k}^{+}$, or aposteriori estimate, with covariance matrix of the estimation error $\mathbf{P}_{k}^{-}$and $\mathbf{P}_{k}^{+}$, respectively. The relationships between them and the measurements are given by [10], [11]

$$
\begin{gathered}
\hat{\mathbf{x}}_{k}^{-}=\mathbf{F}_{k-1} \hat{\mathbf{x}}_{k-1}^{+} \\
\mathbf{P}_{k}^{-}=\mathbf{F}_{k-1} \mathbf{P}_{k-1}^{+} \mathbf{F}_{k-1}^{\prime}+\mathbf{G}_{k-1} \mathbf{Q}_{k-1} \mathbf{G}_{k-1}^{\prime} \\
\hat{\mathbf{x}}_{k}^{+}=\hat{\mathbf{x}}_{k}^{-}+\mathbf{K}_{k}\left(\mathbf{y}_{k}-\mathbf{H}_{k} \hat{\mathbf{x}}_{k}^{-}\right) \\
\mathbf{P}_{k}^{+}=\left(\mathbf{I}-\mathbf{K}_{k} \mathbf{H}_{k}\right) \mathbf{P}_{k}^{-} \\
\mathbf{K}_{k}=\mathbf{P}_{k}^{-} \mathbf{H}_{k}^{\prime}\left(\mathbf{H}_{k} \mathbf{P}_{k}^{-} \mathbf{H}_{k}^{\prime}+\mathbf{R}_{k}\right)^{-1}
\end{gathered}
$$

where Eqs. (11) and (12) are called temporal updates of the estimate and the covariance matrix of the estimation error, respectively; Eqs. (13) and (14) are called observational updates of the estimate and the covariance matrix of the 
estimation error, respectively; and Eq. (15) is called Kalman gain matrix [11]. $\mathbf{A}^{\prime}$ denotes the transpose of $\mathbf{A}$.

At every moment in which the state must be estimated, the steps of temporary update (Eqs. (11) and (12)) and observational update (Ecs. (13) and (14)) have to be carried out. The latter also requires the computation of the Kalman gain matrix (Eq. (15)).

\section{A. Extended Kalman Filter $(E K F)$}

The EKF is an extension of the KF for the case where the state model and/or the relationship between the state and the measurements is non-linear [11]. Eqs. (11) and (13) are reformulated as

$$
\begin{gathered}
\hat{\mathbf{x}}_{k}^{-}=\mathbf{f}_{k-1}\left(\hat{\mathbf{x}}_{k-1}^{+}\right) \\
\hat{\mathbf{x}}_{k}^{+}=\hat{\mathbf{x}}_{k}^{-}+\mathbf{K}_{k}\left(\mathbf{y}_{k}-\mathbf{h}_{k}\left(\hat{\mathbf{x}}_{k}^{-}\right)\right) .
\end{gathered}
$$

All other equations remain the same, but replacing the matrices $\mathbf{F}_{k-1}$ and $\mathbf{H}_{k}$ by a linearization of the equations around the latest estimate of the state.

\section{MOdels to BE COMPARED}

For the formulation of the EKF we consider the following models

- Kinematic I: We consider as states the three position states and the three velocity states; the perturbation is given by the acceleration. It corresponds to the model described in Eq. (4).

- Kinematic II: We consider as states the three position states, the three velocity states and the three acceleration states; the perturbation is given by the jerk. It corresponds to the model described in Eq. (5).

- Dynamic I: We consider as states the three position states and the three velocity states related as in Eq. (8). The perturbation is given by the acceleration $\mathbf{a}_{p}$.

- Dynamic II: Similar to Dynamic I, but incorporating the effect of the $J_{2}$ coefficient (Jeffery's second constant in the description of the Earth's gravitational potential [9]) in $\mathbf{a}_{p}$. The perturbation is given by the remaining terms in $\mathbf{a}_{p}$.

- Dynamic III: Similar to Dynamic I, but adding three aditional states to estimate the non-modeled acceleration. The perturbation is given by the jerk.

- Dynamic IV: Similar to Dynamic II, but adding three aditional states to estimate the non-modeled acceleration. The perturbation is given by the jerk.

In all cases we extend the model by incorporating two additional states to contemplate bias, $b_{k}$, and drift, $\dot{b}_{k}$, according to the measurements model as described in II. We also incorporate an element to the disturbances to model the clock "acceleration", $\ddot{b}_{k}$. We assume that the relation between these states corresponds to a linear system

$$
\left[\begin{array}{l}
b_{k} \\
\dot{b}_{k}
\end{array}\right]=\left[\begin{array}{ll}
1 & T \\
0 & 1
\end{array}\right]\left[\begin{array}{l}
b_{k-1} \\
\dot{b}_{k-1}
\end{array}\right]+\left[\begin{array}{c}
\frac{T^{2}}{2} \\
T
\end{array}\right] \ddot{b}_{k-1} .
$$

Thus, in all cases the linearization of the system function is given by

$$
\mathbf{F}_{k}=\left[\begin{array}{cc}
{\left[\begin{array}{ll}
1 & T \\
0 & 1
\end{array}\right]} & \mathbf{0}_{\mathbf{2} \times \mathbf{M}} \\
\mathbf{0}_{\mathbf{M} \times \mathbf{2}} & \mathbf{F}_{k}^{\star}
\end{array}\right]
$$

where $\mathbf{F}_{k}^{\star}$ denotes the system matrix state without expanding, and $M$ depends on the dimensions of $\mathbf{F}_{k}^{\star}$. E.g., for Kinematic models I and II this matrix is obtained by inspection of Eqs. (4) and (5) (i.e. the leftmost matrix of the right-hand side of each equation); and for the Dynamic I model it's

$$
\begin{aligned}
& \mathbf{F}_{k}^{\star}=\left[\begin{array}{cc}
\left(1-\frac{T^{2}}{2} \frac{\mu}{r_{k}^{3}}\right) \mathbf{I}_{\mathbf{3} \times \mathbf{3}} & T \mathbf{I}_{\mathbf{3} \times \mathbf{3}} \\
-T \frac{\mu}{r_{k}^{3}} \mathbf{I}_{\mathbf{3} \times \mathbf{3}} & \mathbf{I}_{\mathbf{3} \times \mathbf{3}}
\end{array}\right]+ \\
& +\left[\begin{array}{cc}
\frac{T^{2}}{2} \mathbf{S}\left(\boldsymbol{\omega}_{e i}^{e}\right)^{2} & \frac{T^{2}}{2} 2 \mathbf{S}\left(\boldsymbol{\omega}_{e i}^{e}\right) \\
T \mathbf{S}\left(\boldsymbol{\omega}_{e i}^{e}\right)^{2} & T 2 \mathbf{S}\left(\boldsymbol{\omega}_{e i}^{e}\right)
\end{array}\right] .
\end{aligned}
$$

The input matrix is given by

$$
\mathbf{G}_{k}=\left[\begin{array}{cc}
{\left[\begin{array}{c}
\frac{T^{2}}{2} \\
T
\end{array}\right]} & \mathbf{0}_{\mathbf{2} \times \mathbf{1}} \\
\mathbf{0}_{\mathbf{M} \times \mathbf{1}} & \mathbf{G}_{k}^{\star}
\end{array}\right]
$$

where $\mathbf{G}_{k}^{\star}$ denotes the input array for the state without expanding, and $M$ depends on the dimensions of $\mathbf{G}_{k}^{\star}$. For Kinematic I and II $\mathbf{G}_{k}^{\star}$ is obtained by inspection from Eqs. (4) and (5) (i.e. the rightmost matrix of the right-hand side of each equation); for Dynamic I and II, it is equal to those of Kinematic I, while for Dynamic III and IV is equal to those of Kinematic II.

Meanwhile, assembling the vector of measurements as the concatenation of measurements of pseudorange and deltarange

$$
\mathbf{y}_{k}=\left[\begin{array}{ll}
\rho_{k}^{\prime} & \dot{\boldsymbol{\rho}}_{k}^{\prime}
\end{array}\right]^{\prime}
$$

the matrix $\mathbf{H}_{k}$ results

$$
\begin{gathered}
\left.\mathbf{H}_{k} \triangleq \frac{\partial \mathbf{h}\left(\mathbf{x}_{k}\right)}{\partial \mathbf{x}_{k}}\right|_{\hat{\mathbf{x}}_{k}^{-}}= \\
=\left[\begin{array}{ccccc}
\mathbf{1}_{N_{k} \times 1} & \mathbf{0}_{N_{k} \times 1} & \mathbf{H}_{k}^{\star} & \mathbf{0}_{N_{k} \times 3} & \mathbf{0}_{N_{k} \times L} \\
\mathbf{0}_{N_{k} \times 1} & \mathbf{1}_{N_{k} \times 1} & \mathbf{0}_{N_{k} \times 3} & \mathbf{H}_{k}^{\star} & \mathbf{0}_{N_{k} \times L}
\end{array}\right]
\end{gathered}
$$

where $\mathbf{H}_{k}^{\star}$ is a matrix containing in its rows the line-ofsight vectors, Eq. (2), corresponding to the satellites whose measurements were grouped into $\mathbf{y}_{k}, N_{k}$ is the number of measurements available at time $k$, and $L$ is equal to 3 if the state includes acceleration and zero otherwise.

\section{Simulation Results}

To define a significant simulation scenario, we generate the true states of position and velocity that would have our vehicle in a given time interval (actually we are interested in a sampled interval, $t_{k}=k T, k \in K \subset \mathbb{Z}$ ). Based on these states and a description of the GNSS constellation we synthesize the measurements that the vehicle would have at each time $k$. By entering these synthesized measurements to the filtering algorithm (in each of its variants) the estimation of the corresponding states is obtained. In this way, it is possible to compare the solutions obtained in each case with 
the true solution and analyze the performance attained using the different proposed models.

We consider a LEO satellite with orbital parameters $i=98$, $e=0, a=7.028 \times 10^{3} \mathrm{~m}$, similar to the nominal orbital parameters of the argentine SAC-D satellite, a simulation interval equal to the orbital period and a sampling step of 1 second. To generate the states of position and velocity we use the model with disturbances described in section IV in which all disturbances are modeled in detail accordingly to e.g. [8], without considering trust forces.

We also obtain the status of GNSS satellites in the simulation interval as described e.g. in [1], using a set of arbitrarily chosen broadcast ephemeris, and determine which of them are visible by the vehicle at each point of its trajectory.

We next synthesize the pseudorange and deltarange measurements for each one of the satellites in view at every moment. For that, we include a model of the user's clock bias and drift (Eqs. 1 and 3), and the noise affecting each measurement.

We adjust the covariance matrix $\mathbf{R}_{k}$ (used in Eq. (15)) with the same value used to generate the measurements' noise, and compare the performance of each filtering scheme (with the diverse proposed models) for different values of the noise model covariance matrix $\mathbf{Q}_{k}$ (used in Eq. (12)), given by

$$
\mathbf{Q}_{k}=\left[\begin{array}{cc}
\sigma_{\ddot{b}}^{2} & \mathbf{0}_{1 \times 3} \\
\mathbf{0}_{1 \times 3} & \sigma_{\alpha}^{2} \mathbf{I}_{3 \times 3}
\end{array}\right]
$$

where $\sigma_{\alpha}=\sigma_{\ddot{x}}$ for those models in which it is considered that the disturbance is given by the acceleration, and $\sigma_{\alpha}=\sigma_{j}$ for those models where it is considered that the perturbation is given by the jerk. To analyze the performance of each of the models we used as a metric the mean square error in biasposition and drift-velocity solutions

$$
\begin{aligned}
& \sqrt{\overline{\overline{\epsilon_{p}^{2}}}}=\sqrt{\frac{1}{K} \sum_{k=0}^{K-1} \frac{\left\|\hat{\mathbf{x}}_{p, k}-\mathbf{x}_{p, k}\right\|^{2}}{4}} \\
& \sqrt{\overline{\bar{\epsilon}_{v}^{2}}}=\sqrt{\frac{1}{K} \sum_{k=0}^{K-1} \frac{\left\|\hat{\mathbf{x}}_{v, k}-\mathbf{x}_{v, k}\right\|^{2}}{4}}
\end{aligned}
$$

where $\mathbf{x}_{p, k}$ and $\hat{\mathbf{x}}_{p, k}$ respectively denote the sub-vectors of $\mathbf{x}_{k}$ and $\hat{\mathbf{x}}_{k}$, that contain the three position coordinates and bias (in meters) elements; and $\mathbf{x}_{v, k}$ and $\hat{\mathbf{x}}_{v, k}$ respectively denote the sub-vectors of $\mathbf{x}_{k}$ and $\hat{\mathbf{x}}_{k}$ that contain the three velocity coordinates and drift (in $\mathrm{m} / \mathrm{s}$ ) elements.

In Figs. 1 and 2 the results of evaluation of these metrics as a function of the parameter $\sigma_{\ddot{x}}$ (or $\sigma_{j}$ ), for the different models proposed, are presented. The optimal values are summarized in Table I. In this case GPS measurements are used. The results obtained with the GLONASS system are similar.

It can be seen that, when tuning the filter with small values of "sigmas", the estimation error is large. This tuning can be interpreted as putting more confidence in the model than in the measurements; however, if the model is not quite accurate, putting too much confidence in it leads to bigger estimation

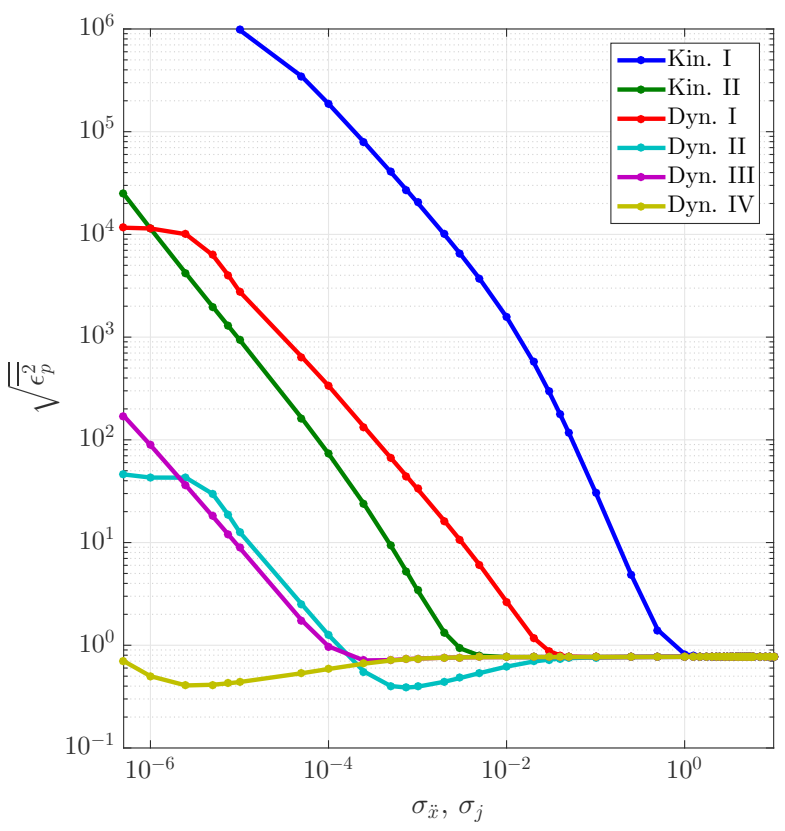

Fig. 1. Mean square error in bias-position solutions as a function of $\sigma_{\ddot{x}}$ (or $\sigma_{j}$ ) for the six models considered using GPS system. In all cases $\sigma_{\ddot{b}}=0.01$ is taken.

TABLE I

RESULTS OF COMPARISON OF DIFFERENT MODELS.

\begin{tabular}{|c|c|c|c|c|}
\hline Mod. & pt. & $\sigma_{\ddot{x}}\left(\sigma_{j}\right)$ & $\sqrt{\mathrm{E}\left\{\epsilon_{p}^{2}\right\}}$ & $\sqrt{\mathrm{E}\left\{\epsilon_{v}^{2}\right\}}$ \\
\hline \multirow{2}{*}{ Kin. I } & Pos & 5.75 & $7.71 \times 10^{-1}$ & $1.56 \times 10^{-1}$ \\
& Vel & 6.00 & $7.71 \times 10^{-1}$ & $1.56 \times 10^{-1}$ \\
\hline \multirow{2}{*}{ Kin. II } & Pos & $2.00 \times 10^{-2}$ & $7.68 \times 10^{-1}$ & $9.69 \times 10^{-2}$ \\
& Vel & $2.00 \times 10^{-2}$ & $7.68 \times 10^{-1}$ & $9.69 \times 10^{-2}$ \\
\hline \multirow{2}{*}{ Dyn. I } & Pos & $1.00 \times 10^{-1}$ & $7.65 \times 10^{-1}$ & $8.64 \times 10^{-2}$ \\
& Vel & $4.00 \times 10^{-2}$ & $7.98 \times 10^{-1}$ & $6.85 \times 10^{-2}$ \\
\hline \multirow{2}{*}{ Dyn. II } & Pos & $7.50 \times 10^{-4}$ & $3.87 \times 10^{-1}$ & $1.69 \times 10^{-2}$ \\
& Vel & $7.50 \times 10^{-4}$ & $3.87 \times 10^{-1}$ & $1.69 \times 10^{-2}$ \\
\hline \multirow{2}{*}{ Dyn. III } & Pos & $2.50 \times 10^{-4}$ & $7.12 \times 10^{-1}$ & $3.20 \times 10^{-2}$ \\
& Vel & $2.50 \times 10^{-4}$ & $7.12 \times 10^{-1}$ & $3.20 \times 10^{-2}$ \\
\hline \multirow{2}{*}{ Dyn. IV } & Pos & $2.50 \times 10^{-6}$ & $4.06 \times 10^{-1}$ & $1.57 \times 10^{-2}$ \\
& Vel & $2.50 \times 10^{-6}$ & $4.06 \times 10^{-1}$ & $1.57 \times 10^{-2}$ \\
\hline
\end{tabular}

errors. In other words, the estimator cannot follow the user's variations, resulting in estimation errors.

On the other hand, tuning the filter to large values of "sigmas" also leads to large estimation errors. This tuning can be interpreted as relying more in the measurements than in the model; however, since the measurements are contaminated with noise, too much reliance on them leads to the noise not being filtered. In other words, the estimator follows the noiseproduced variations of the measurements, resulting again in estimation errors.

It can be seen that the Kinematic I model is the one with the worst performance, which is expected based on its simplicity: it does not attempt to model or estimate the acceleration. It may also be seen that Kinematic II and Dynamic I models 


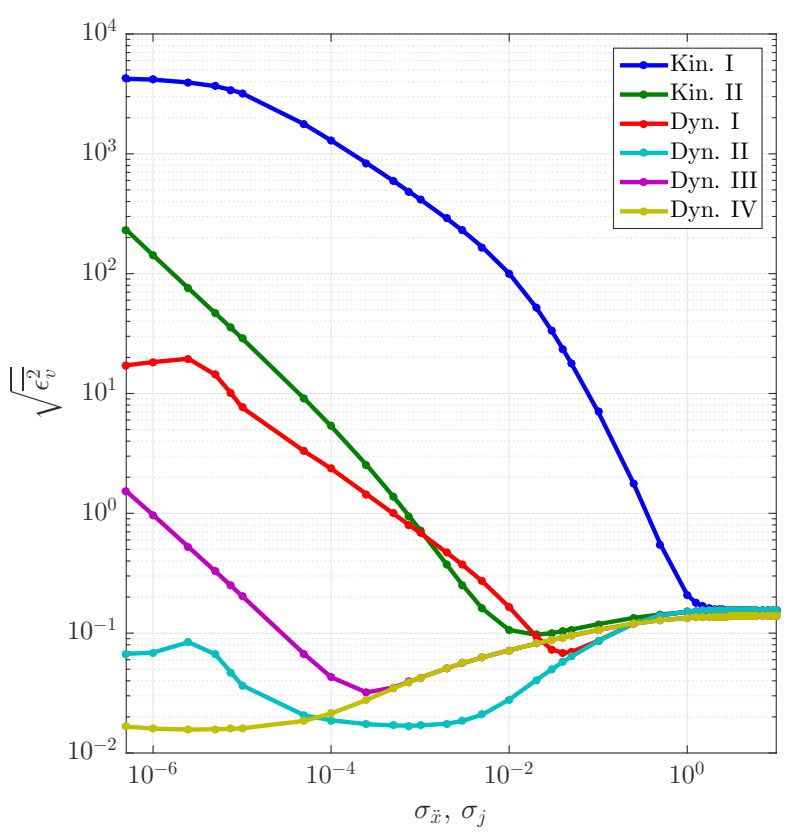

Fig. 2. Mean square error in drift-velocity solutions as a function of $\sigma_{\ddot{x}}$ (or $\sigma_{j}$ ) for the six models considered using GPS system. In all cases $\sigma_{\ddot{b}}=0.01$ is taken.

have a similar performance, i.e. using a simple acceleration model achieves a similar performance to trying to estimate it on the filter.

It may also be noted that the Dynamic III model has a better performance, since that, besides using a simple model of acceleration, it estimates the non-modeled acceleration. Finally, Dynamic II and IV models are the ones that get the best performance, which is expected because they use a more accurate model of acceleration including the effect of $J_{2}$. The fact that both obtain a similar performance is because the effect of non-modeled acceleration is small in this case and therefore, estimating it doesn't produce any advantage over leaving it as part of the unmodelled perturbations.

Figures 1 and 2 are also useful for analyzing how the performance of each model gets worse in case of choosing another tuning value, e.g. when trying to obtain a better performance against non-ideal effects, which have not been taken into account.

In comparing the Kinematic II and Dynamic III and IV models with others it should be noted that the horizontal axis corresponds to jerk, while in the others it corresponds to acceleration.

Searching the optimal considering the $\sigma_{\ddot{b}}$ dimension as well (not presented due to space limitations), shows that the optimal value is obtained for $\sigma_{\ddot{b}}=0.01$ or very close values. In the latter case, the optimum value doesn't differ significantly from that obtained taking $\sigma_{\ddot{b}}=0.01$. For this reason this value was used in the comparison of all models.

\section{APPLICATION EXAMPLE}

As a real-data application example, we analyzed GPS measurements taken on board the argentine SAC-D satellite. In this case we applied corrections to systematic errors in the pseudorange and deltarange measurements, and we model the standard deviation for each measurement according to the $C / N_{0}$ estimated by the receiver itself. Since there are not real positions and velocities of reference, we chose to compare the results obtained with different models among themselves.

In Figs. 3 and 4 errors observed in the three coordinates of position and velocity using the Dynamic II model, with reference to the solution of the Dynamic IV model are presented. In order to make a comparison, the errors against the same reference obtained in the punctual solution (an algebraic solution based on the measurements sampled simultaneusly on a single time instant) are also presented. To this end, we consider the same noise covariance matrix of measurements used in the filtering process. The $\pm 3 \sigma$ error intervals, based on the corresponding element on $\mathbf{P}_{k}^{+}$, are also plotted in each coordinate. It can be seen that virtually at all times the error is kept within these limits.

When comparing the results obtained with other models, a good correspondence with simulation results was observed. This allows a real data validation of the operation of the proposed filtering schemes.
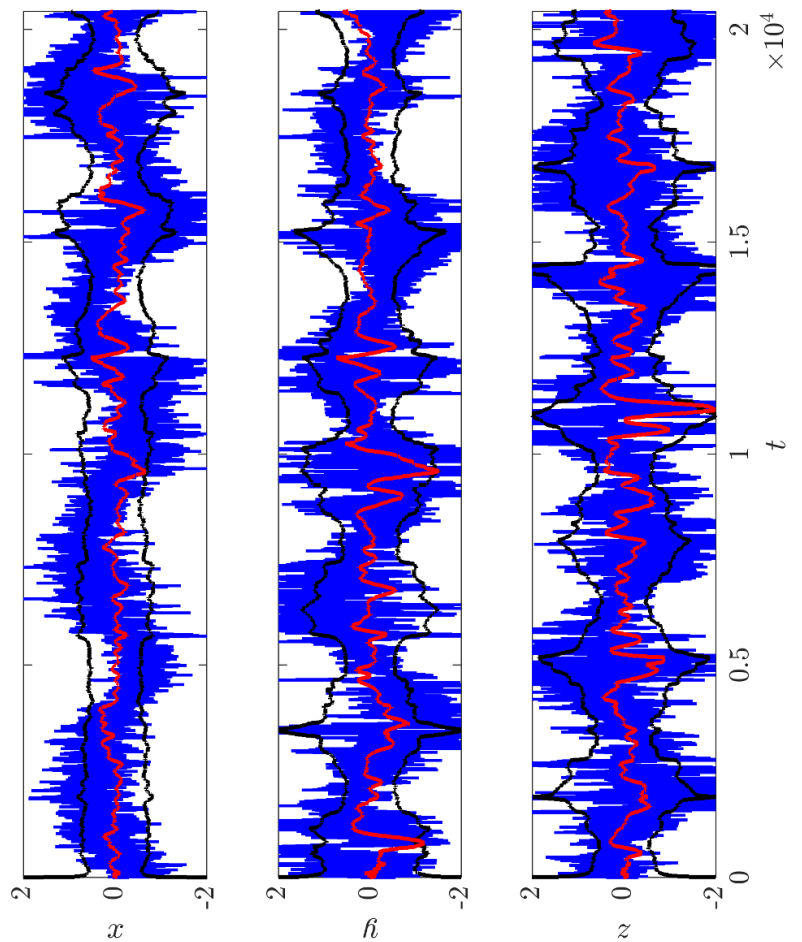

Fig. 3. Error in solving point position (blue) and by EKF (red) using the Dynamic II model, with respect to the solution taken as a reference. The interval $\pm 3 \sigma$ of error estimated by the same filter (black) is also displayed. 

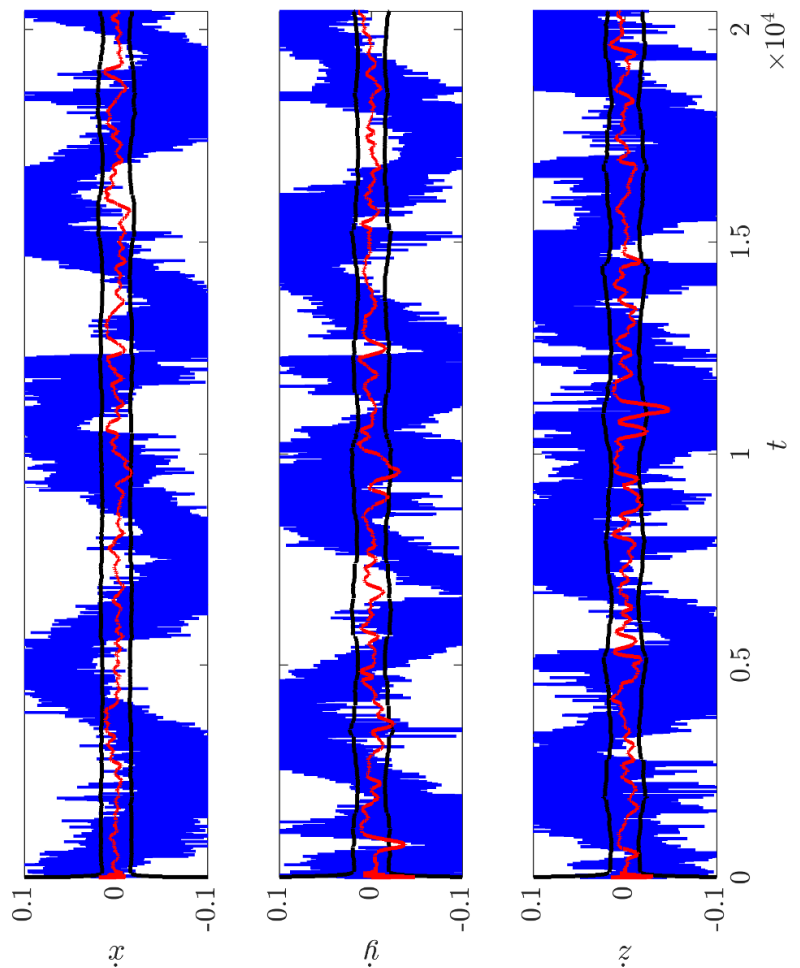

Fig. 4. Error in solving point velocity (blue) and by EKF (red) using the Dynamic II model, with respect to the solution taken as a reference. The interval $\pm 3 \sigma$ of error estimated by the same filter (black) is also displayed.

\section{CONCLUSIONS}

In this work we proposed different statistical filtering models that can be employed in the formulation of an EKF filtering scheme for LEO satellite navigation based on GNSS measurements. The simplest ones are based on a kinematic approach and could be extended to a variety of vehicles. The other ones are based on a dynamic approach that takes into account the most significant forces acting in the motion of this kind of vehicle. It should be noted that in all cases we prioritize obtaining simple models, feasible for implementing real-time algorithms, i.e. without a high computational burden associated.

Based on a representative simulation scenario, we compared the performance of these different models obtaining in each case the optimum tuning value of the filter and analyzing the relative improvements of each one. Based on actual measurements took by the argentine satellite SAC-D, the applicability of filtering schemes proposed for this kind of vehicle was also verified.

From a purely qualitative analysis, we can conclude that, in general the better performance is attained with the most complex implementation in terms on computational burden. However, the additional complexity seems not to be prohibitive for a real-time implementation. A quantitative analysis of the computational burden depends on the particular architecture in which the algorithms will be implemented and it's beyond the scope of this work.

As future work, an analysis of the performance against unmodeled disturbances, such as the effect of thrust, will be addressed. Also, some modifications needed in order to use measurements from more than one GNSS system, i.e. GLONASS or Galileo, will also be considered.

\section{ACKNOWLEDGMENT}

This work was funded by the Argentine ANPCyT grant PICT2014-1232, Universidad Nacional de La Plata grant 11I-166, and CIC Pcia de Buenos Aires.

Real data used in this work were provided by the CONAE (Comisión Nacional de Actividades Espaciales) within the framework of the Satellite SAC-D/Aquarius mission.

\section{REFERENCES}

[1] E. Kaplan and C. Hegarty, Understanding GPS: Principles and Applications. Artech House, 2005.

[2] B. Hofmann-Wellenhof, H. Lichtenegger, and E. Wasle, GNSS - Global Navigation Satellite Systems: GPS, GLONASS, Galileo, and more. Springer, 2007.

[3] O. Montenbruck, T. van Helleputte, R. Kroes, and E. Gill, "Reduced dynamic orbit determination using GPS code and carrier measurements," Aerospace Science and Technology, vol. 9, no. 3, pp. 261 - 271, 2005.

[4] H. Bock, A. Jäggi, D. Švehla, G. Beutler, U. Hugentobler, and P. Visser, "Precise orbit determination for the GOCE satellite using GPS," Advances in Space Research, vol. 39, no. 10, pp. 1638 - 1647, 2007.

[5] E.-J. Choi, J.-C. Yoon, B.-S. Lee, S.-Y. Park, and K.-H. Choi, "Onboard orbit determination using GPS observations based on the unscented kalman filter," Advances in Space Research, vol. 46, no. 11, pp. 1440 1450, 2010.

[6] P. C. Pinto, M. Pardal, H. K. Kuga, and R. V. de Moraes, "A discussion related to orbit determination using nonlinear sigma point kalman filter," Mathematical Problems in Engineering, 2009.

[7] J. Cogo, J. G. García, R. P.A., and C. H. Muravchik, "Comparación de Métodos de Filtrado Estadístico para Navegación de Satélites LEO con Señales GNSS," in Argencon 2014, 2014.

[8] O. Montenbruck and E. Gill, Satellite Orbits: Models, Methods and Applications. Springer Berlin Heidelberg, 2012.

[9] A. Tewari, Atmospheric and Space Flight Dynamics: Modeling and

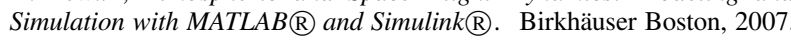

[10] M. Grewal and A. Andrews, Kalman filtering: theory and practice using $M A T L A B$, 2nd ed. Wiley, 2001.

[11] D. Simon, Optimal State Estimation: Kalman, H Infinity, and Nonlinear Approaches. Wiley, 2006. 